\title{
Sevoflurane inhibits migration and invasion of colorectal cancer cells by regulating microRNA-34a/ADAM10 axis
}

\author{
S. Q. SUN, L. J. REN, J. LIU, P. WANG, S. M. SHAN* \\ Department of Anesthesiology, the Fifth Central Hospital of Tianjin, Tianjin, China \\ *Correspondence: shanbzgxian@126.com
}

Received December 13, 2018 / Accepted April 10, 2019

\begin{abstract}
Sevoflurane is frequently used volatile anesthetic in cancer surgery. It has been suggested that treatment with sevoflurane could suppress migration and invasion of several human cancer cells in vitro. However, the effects of sevoflurane on colorectal cancer (CRC) remain largely unclear. In this study, CRC HCT116 and SW480 cells were treated by various concentrations of sevoflurane. MTT assay and Transwell assay were applied to evaluate the cell viability, migration and invasion abilities of CRC cell lines, respectively. Real-time quantitative PCR (RT-qPCR) was used to examine the expression level of miR-34a, and western blot assay was employed to detect the protein level of ADAM10. The target interaction between miR-34a and ADAM10 was verified through bioinformatics analysis and luciferase reporter gene assay system. Aberrant inhibitory effects induced by sevoflurane on the cell viability, migration and invasion abilities of HCT116 and SW480 cells in a dose-dependent manner were observed. Upregulation of miR-34a strikingly suppressed the cell proliferation, migration and invasion abilities of the two cell lines. Sevoflurane could facilitate the miR-34a expression and its suppressive effects on CRC cells were reversed by pre-treatment with miR-34a inhibitors. ADAM10 was identified as a downstream gene of miR-34a, downregulated by miR-34a. Overexpression of ADAM10 reverted both miR-34a and sevoflurane-induced repression in the cell proliferation, migration and invasion abilities of CRC cells. Our data showed sevoflurane inhibits the migration and invasion of colorectal cancer cells by regulating microRNA-34a/ADAM10 axis.
\end{abstract}

Key words: colorectal cancer, sevoflurane, miR-34a, ADAM10, migration and invasion

Colorectal cancer (CRC) is a common malignant tumor, leading in both morbidity and mortality worldwide [1]. A prior report indicated that about $6 \times 10^{5}$ people die of CRC each year [2]. Nowadays, the main approaches of CRC therapy are surgery, radiation therapy and chemotherapy, that is still the mainstay of therapy for advanced CRC [3]. Though the treatment methods of CRC have been elevated over the decades, there is still a long way for the investigation of CRC diagnosis or treatment.

Sevoflurane is an inhalational anesthetic gas that is commonly used in numerous surgical operations of cancer patients [4]. It has been proved that sevoflurane could inhibit cell growth, invasion and migration, trigger morphologic changes and apoptosis in several types of cancer cell lines [4-7].

MicroRNAs (miRNAs) are a class of small non-coding RNA that specifically repress the translation and trigger the degradation of mRNAs, control genes participating in cellular processes such inflammation, cell cycle regulation, stress response, differentiation, apoptosis, migration and invasion [8,9]. Mounting evidence has suggested that miRNAs are involved in influencing the cell proliferation, migration or invasion ability in CRC cells. A previous study demonstrated that upregulation of miR-124 in CRC cells restrained cell growth and colony formation ability [10]. The study conducted by Zhang et al. verified that miR-384 could effectively suppress the proliferation of CRC cells by direct targeting AKT3 [11]. On the contrary, Zhang et al. proved miR-182 promotes CRC cell growth and invasion, and its contribution is associated to forkhead box F2 transcription factor [12]. Also, it has been demonstrated that exogenous introduction of miR-92a could promote cell proliferation, migration and invasion of CRC LoVo and SW480 cells [13]. The miR-34 family comprises of miR-34a, miR-34b and $\mathrm{miR}-34 \mathrm{c}$, and permanent inactivation of the members of the miR-34 family induced cell cycle arrest, senescence and apoptosis of tumor cells [14]. Accumulating studies demonstrated that dysregulation of miR-34a was involved in cell cycle, proliferation, migration or invasion, functioning as a tumor suppressor in prostate cancer, hepatocellular 
carcinoma (HCC), gastric cancer, esophageal cancer and colorectal cancer [15-19].

The ADAMs (a disintegrin and metalloproteinase) is a group of transmembrane and secreted proteins with various biological roles. An emerging body of evidence has indicated that ADAMs mediated angiogenesis, neurogenesis as well as heart development and are involved in cancer growth, invasion and metastasis $[20,21]$. ADAM10 has been revealed to be related to tumor formation and cancer cell proliferation, migration and invasion [22]. ADAM10 also functions as a potential cancer biomarker for lung cancer [23]. The single nucleotide polymorphisms (SNPs) of ADAM10 are correlated with HCC progression, and may serve as therapeutic targets to examine prognosis of HCC patients [24].

In the present study, we aimed to examine the role of sevoflurane on the cell viability, migration and invasion abilities of CRC HCT116 and SW480 cells and explore the underlying mechanism.

\section{Materials and methods}

Cell culture and sevoflurane treatment. CRC cell lines HCT116 and SW480 were obtained from American Type Culture Collection (ATCC, Manassas, VA, USA). Cells were cultured in Roswell Park Memorial Institute 1640 medium (RPMI1640; Gibco, Grand Island, NY, USA) supplemented with $10 \%$ fetal bovine serum (FBS; HyClone, Logan, UT, USA) at $37^{\circ} \mathrm{C}$ with $5 \% \mathrm{CO}_{2}$ humidified atmosphere.

Cells were exposed to various concentrations of sevoflurane with a mixture of $95 \%$ air and $5 \% \mathrm{CO}_{2}$ at $6 \mathrm{l} / \mathrm{min}$ for 6 $\mathrm{h}$ as previously described $[6,25]$. The HCT116 and SW480 cells were divided into 4 groups: control group (without sevoflurane), $1.7 \%$ sevoflurane group, $3.4 \%$ sevoflurane group and $5.1 \%$ sevoflurane group. To investigate whether sevoflurane suppresses the cell viability and metastasis of CRC cells by targeting miR-34a or ADAX10, additional groups of HCT116 and SW480 cells were treated with miR-34a inhibitor, pcDNA3.1-ADAX10 or their respective negative control for $48 \mathrm{~h}$ prior to exposure to a concentration at $5.1 \%$ sevoflurane. Cells at the logarithmic phase were seeded onto plates and incubated for $24 \mathrm{~h}$ at $37^{\circ} \mathrm{C}$. The cell culture plates were then placed in an airtight glass chamber connected to an anesthesia device (Cicero-EM 8060; Drager, Lübeck, Germany) at the inlet port and the other port of chamber was a gas monitor (PM8060; Drager) to monitor the concentrations of sevoflurane. The anesthesia machine was attached to an anesthetic vaporizer (Sevorane; Abbott, Abbot Park, IL, USA) to supply sevoflurane. After the treatment with sevoflurane, the cells were incubated for another $24 \mathrm{~h}$ at $37^{\circ} \mathrm{C}$ and then used for MTT and Transwell assay.

MTT assay. $2 \times 10^{3}$ HCT116 and SW480 cells were seeded onto 96-well plates with RPMI1640 medium and incubated at $37^{\circ} \mathrm{C}$ for $24 \mathrm{~h}$. Then $20 \mu \mathrm{l}$ 3-(4,5-dimethylthiazol-z-yl)-2,5diphenyltetrazolium bromide (MTT, $5 \mathrm{mg} / \mathrm{ml}$; Beyotime, Shanghai, China) were added into each well and incubated for another $4 \mathrm{~h}$ at $37^{\circ} \mathrm{C}$. Then the supernatant was removed, and $150 \mu \mathrm{l}$ dimethyl sulfoxide (DMSO, Sigma-Aldrich, St. Louis, MO, USA) were added to each well. The absorbance was measured at $490 \mathrm{~nm}$ using a microplate reader (Bio-Rad, Hercules, CA, USA). Three independent experiments were performed.

Transwell assay. Invasion assay was carried out using Transwell chamber $(8 \mu \mathrm{m}$ pores; BD Biosciences, San Jose, CA, USA) as previously described [7]. Briefly, $1 \times 10^{4}$ HCT116 and SW480 cells in $100 \mu \mathrm{l}$ RPMI1640 medium without FBS were seeded in the upper chamber with matrigel $(24 \mu \mathrm{g} / \mu \mathrm{l}$; R\&D Systems, Inc., Minneapolis, MN, USA) added on the top of upper chamber in advance. Then $500 \mu$ medium with $10 \%$ FBS was added to the lower chamber as a chemoattractant, incubated for $8 \mathrm{~h}$ at $37^{\circ} \mathrm{C}$. Then the insert was washed three times with phosphate-buffered saline, and the cells on the upper surface of the membrane were wiped off using a cotton swab. The cells on the lower surface were fixed with methanol, stained with $0.1 \%$ crystal violet (Sigma-Aldrich) and counted under a microscope $(\times 100$ magnification) (Olympus, Tokyo, Japan) in five random fields.

The protocol of migration assay was similar to the measurement of invasion assay, except the matrigel on the membrane. All measurements were run in triplicate.

RT-qPCR assay. Total RNA was isolated from HCT116 and SW480 cells using Trizol reagent (Invitrogen, Carlsbad, CA, USA). Reverse transcription was performed with a PrimeScript RT Reagent kit (Takara Bio, Inc., Otsu, Japan) from $500 \mathrm{ng}$ total RNA template. Expression of miR-34a was assessed with qPCR using SYBR Green Real-Time PCR Master Mix (Roche Diagnostics, Basel, Switzerland) with Applied Biosystems 7500 thermocycler (Thermo Fisher Scientific, Inc., Waltham, MA, USA). U6 was used as control. The primers for miR-34a and U6 were purchased from Ambion (Austin, TX, USA). All experiments were performed for three times. The data were calculated using the threshold cycle $2^{\Delta \Delta \mathrm{Ct}}$ method.

Transfection of HCT116 and SW480 cells. miR-34a mimics, miR-NC mimics, miR-34a inhibitors or miR-NC inhibitors were obtained from GenePharma Co. Ltd. Shanghai, China. The pcDNA 3.1 plasmid was purchased from Thermo Fisher Scientific and pcDNA 3.1-ADAM10 plasmid was conducted and stored in our laboratory. Above oligonucleotides or plasmids were transfected into HCT116 and SW480 cells using Lipofectamine ${ }^{\mathrm{Tm}} 2000$ reagent (Invitrogen,) referring to the protocols of manufacturer.

Luciferase assays. The online TargetScan (http://www. targetscan.org) was used to predict the downstream genes of miR-34a. ADAX10 was identified as a potential target of miR-34a. Then luciferase reporter gene plasmid ADAM10WT or ADAM10-MUT were constructed with pGL3 luciferase promoter vector (pGL3-empty, Promega, Madison, WI, USA). HCT116 and SW480 cells were seeded at $2 \times 10^{5}$ cells per well in a 24-well plate and were co-transfected with ADAM10-WT, ADAM10-MUT, and miR-34a mimics or 
miR-NC mimics using Lipofectamine ${ }^{\mathrm{rm}} 2000$ reagent for $48 \mathrm{~h}$. The luciferase activities were analyzed with a Dual-Luciferase Reporter Assay System (Promega) following the manufacturer's protocols. Each experiment was repeated three times.

Western blot assay. HCT116 and SW480 cells were lysed with ice-cold Radio-Immunoprecipitation Assay (RIPA) buffer (Beyotime). Concentration of protein was determined by bicinchoninic acid assay (BCA) protein assay kit (Sigma-Aldrich). Then equal amounts of the protein were separated by $10 \%$ sodium dodecyl sulfate (SDS)-polyacrylamide gels, transferred onto polyvinylidene fluoride (PVDF) membranes (Millipore, Billerica, MA, USA), which were blocked in 5\% non-fat dried milk for $2 \mathrm{~h}$ at room temperature. The PVDF membranes then were incubated overnight at $4^{\circ} \mathrm{C}$ with primary antibodies against ADAM10 (1:1000 dilution) and GAPDH (1:2000 dilution). After being washed three times with PBS, the membranes were incubated with HRP-conjugated secondary antibodies (1:2000 dilution) at room temperature for $2 \mathrm{~h}$. Immunoreactive bands were visualized by an enhanced chemiluminescence procedure (ECL, Thermo Fisher Scientific) and level of protein expression was analyzed using Quantity One software (Bio-Rad Laboratories). All antibodies were purchased from Cell Signaling Technology (Danvers, MA, USA).

Statistical analysis. All data analyses were conducted using SPSS 18.0 software (SPSS, Chicago, IL, USA). All values were exhibited as mean \pm SEM. Student's t-test and LSD-t were used to analyze difference in 2 groups and 3 or more groups data respectively. A p-value $<0.05$ was defined as statistically significant.
A

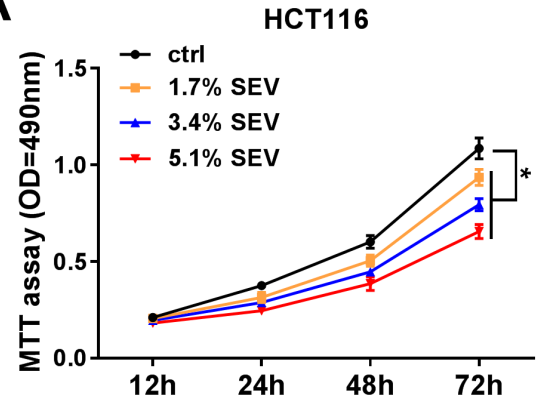

B

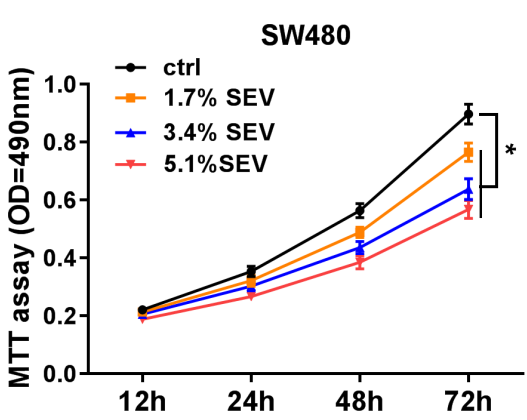

C

C.
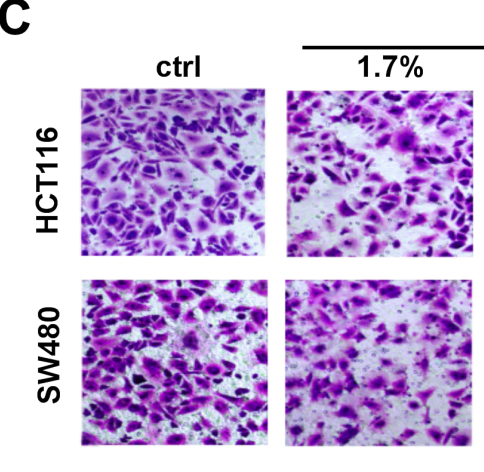

sevoflurane

D
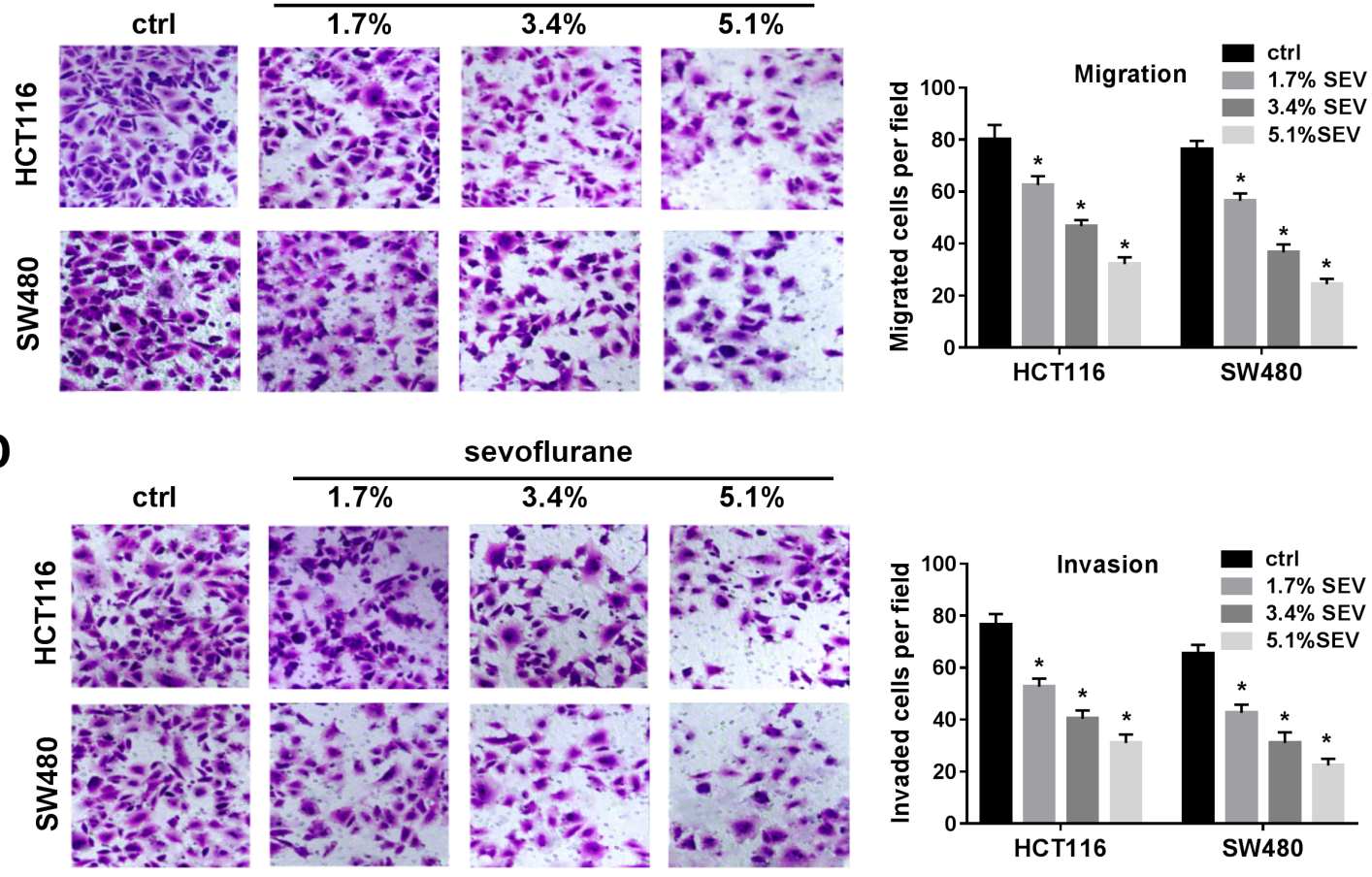

Figure 1. Sevoflurane inhibited the cell viability, migration and invasion abilities of CRC cells. A and B) MTT assay was used to manifest the cell viability of HCT116 or SW480 cells at $12 \mathrm{~h}, 24 \mathrm{~h}, 48 \mathrm{~h}$ and $72 \mathrm{~h}$ after treatment with various concentrations of sevoflurane. C and D) Transwell assay was applied to evaluate migration and invasion abilities of HCT116 and SW480 cells after treatment with sevoflurane. ${ }^{*}$ p $<0.05$, ctrl (without treatment). 


\section{Results}

Sevoflurane inhibited the cell viability, migration and invasion abilities of CRC cells. To validate the function of sevoflurane on the progression of CRC cells, MTT assay was employed to detect the cell viability of HCT116 or SW480 cells treated with various concentrations of sevoflurane. Compared with the ctrl group, the HCT116 and SW480 cells treated with sevoflurane showed an obviously decreased cell viability in a concentration-dependent way (Figures 1A and 1B). For cell migration and invasion assay, Transwell assay was applied to measure the migration and invasion abilities of HCT116 or SW480 cells treated with various concentrations of sevoflurane following $8 \mathrm{~h}$ of incubation. We found that treatment with sevoflurane weakened the cell migration and invasion abilities compared with the control group in a concentration-dependent manner (Figures 1C and 1D).

Overexpression of miR-34a repressed cell viability, migration and invasion in CRC cells in vitro. To investigate the effects of sevoflurane on miR-34a expression, we perform RT-qPCR assay to examine the expression of miR-34a in HCT116 and SW480 cells after treatment with various concentrations of sevoflurane and observed that the expression of miR-34a was increased compared with the control group in a concentration-dependent manner (Figure 2A). Then we conducted HCT116 and SW480 cells that miR-34a was upregulated by transfection with miR-34a mimics and RT-qPCR assay was carried out to verify transfection efficiency (Figure 2B). The cell viability was detected through MTT assay and results indicated that overexpression of miR-34a induced an obvious repression of cell viability in the two cell lines (Figures 2C and 2D). As shown in Figures 2E and $2 \mathrm{~F}$, the migration and invasion abilities of HCT116 and SW480 cells were notably blocked by miR-34a mimics.

Sevoflurane suppressed the cell viability, migration and invasion abilities by targeting miR-34a. To explore the mechanism of sevoflurane in progress of CRC cells, we transfected HCT116 and SW480 cells with miR-NC inhibitors or miR-34a inhibitors prior to exposure to a concentration of $5.1 \%$ sevoflurane. Results showed that miR-34a inhibitors significantly reversed the promotion of miR-34a expression triggered by sevoflurane treatment (Figure 3A); miR-34a inhibitors also rescued the repressive effect of sevoflurane on cell viability, migration and invasion in both HCT116 and SW480 cells (Figures 3B-3E).

ADAM10 is a direct target of miR-34a and negatively regulated by miR-34a. TargetScan 7.2 was employed to identify the potential targets of miR-34a. Then we found that the 3'-UTR of ADAM10 mRNA contains putative a binding site for miR-34a (Figure 4A). Following luciferase activity assay was carried out to further confirm the target relationship between miR-34a and ADAM10. Results demonstrated that the luciferase activity of ADAM10-WT was inhibited by miR-34a mimics (Figures 4B and 4C) and elevated by miR-34a inhibitors in both HCT116 and SW480 cells (Figures 4D and $4 \mathrm{E})$. No distinct change of ADAM10-MUT luciferase activity
A

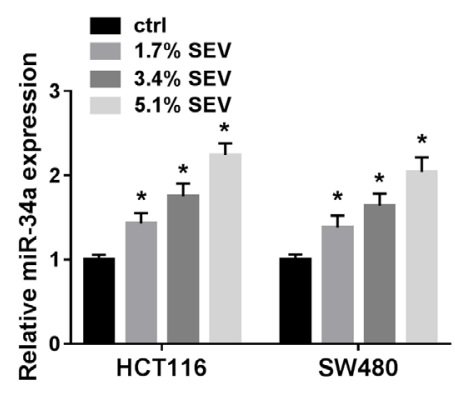

D

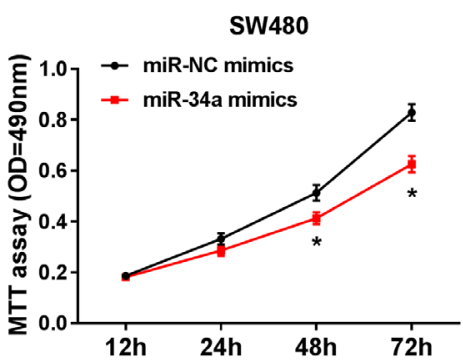

B

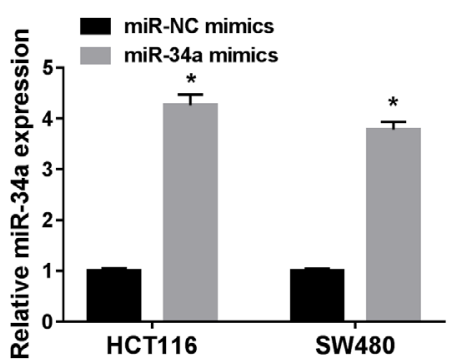

E

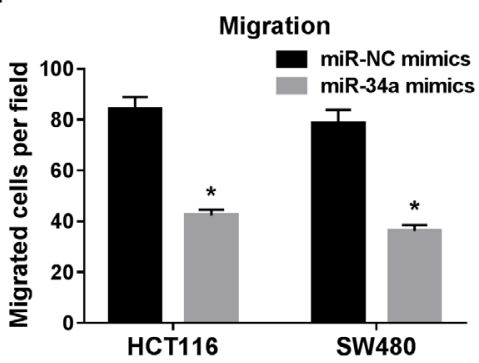

C

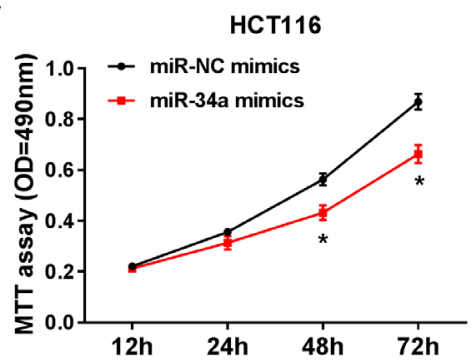

$\mathbf{F}$

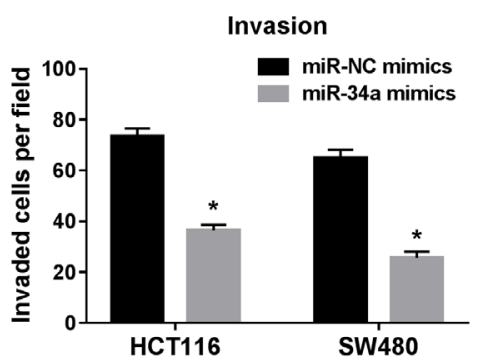

Figure 2. Overexpression of miR-34a repressed cell viability, migration and invasion in CRC cells in vitro. A) The mRNA expression level of miR-34a of HCT116 and SW480 cells after treatment with sevoflurane was detected by RT-qPCR assay. HCT116 and SW480 cells were transfected with miR-NC mimics or miR-34a mimics. B) The miR-34a expression was tested via RT-qPCR assay at $48 \mathrm{~h}$ post transfection in HCT116 and SW480 cells. C and D) The cell viability of HCT116 and SW 480 cells was measured by MTT assay at $12 \mathrm{~h}, 24 \mathrm{~h}, 48 \mathrm{~h}$ and $72 \mathrm{~h}$ after transfection. E and F) The number of migration and invasion cells was counted at $48 \mathrm{~h}$ following transfection in Transwell chamber experiment. ${ }^{\star} \mathrm{p}<0.05$, ctrl (without treatment). 
A

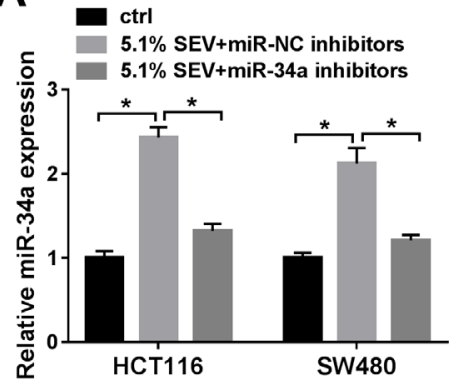

D

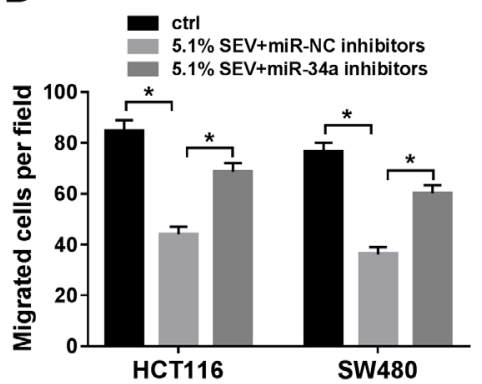

B

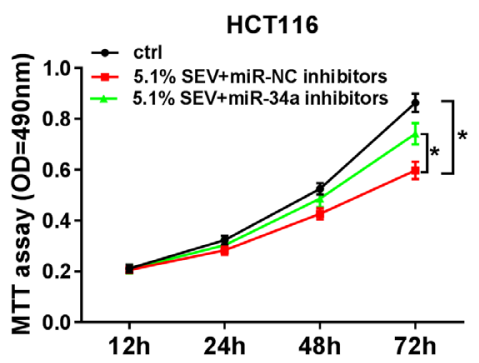

E

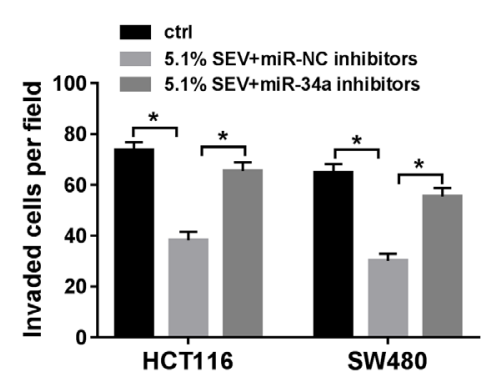

C

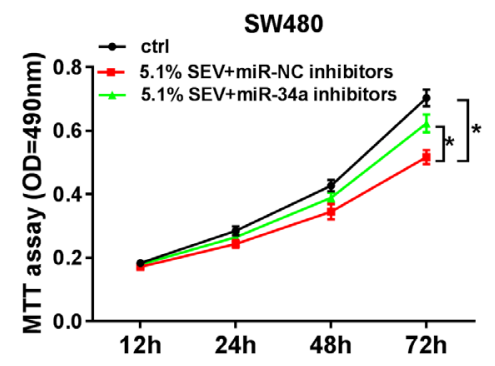

Figure 3. Sevoflurane suppressed the cell viability, migration and invasion abilities by targeting miR-34a. A) RT-qPCR assay showed the expression level of miR-34a in HCT116 and SW480 cells after treatment with sevoflurane and miR-NC inhibitors or miR-34a inhibitors. B and C) MTT assay showed cell viability of HCT116 and SW480 cells after treatment. D and E) Transwell assay exhibited number of migration and invasion cells after treatment. ${ }^{*} \mathbf{p}<0.05$, ctrl (without treatment).
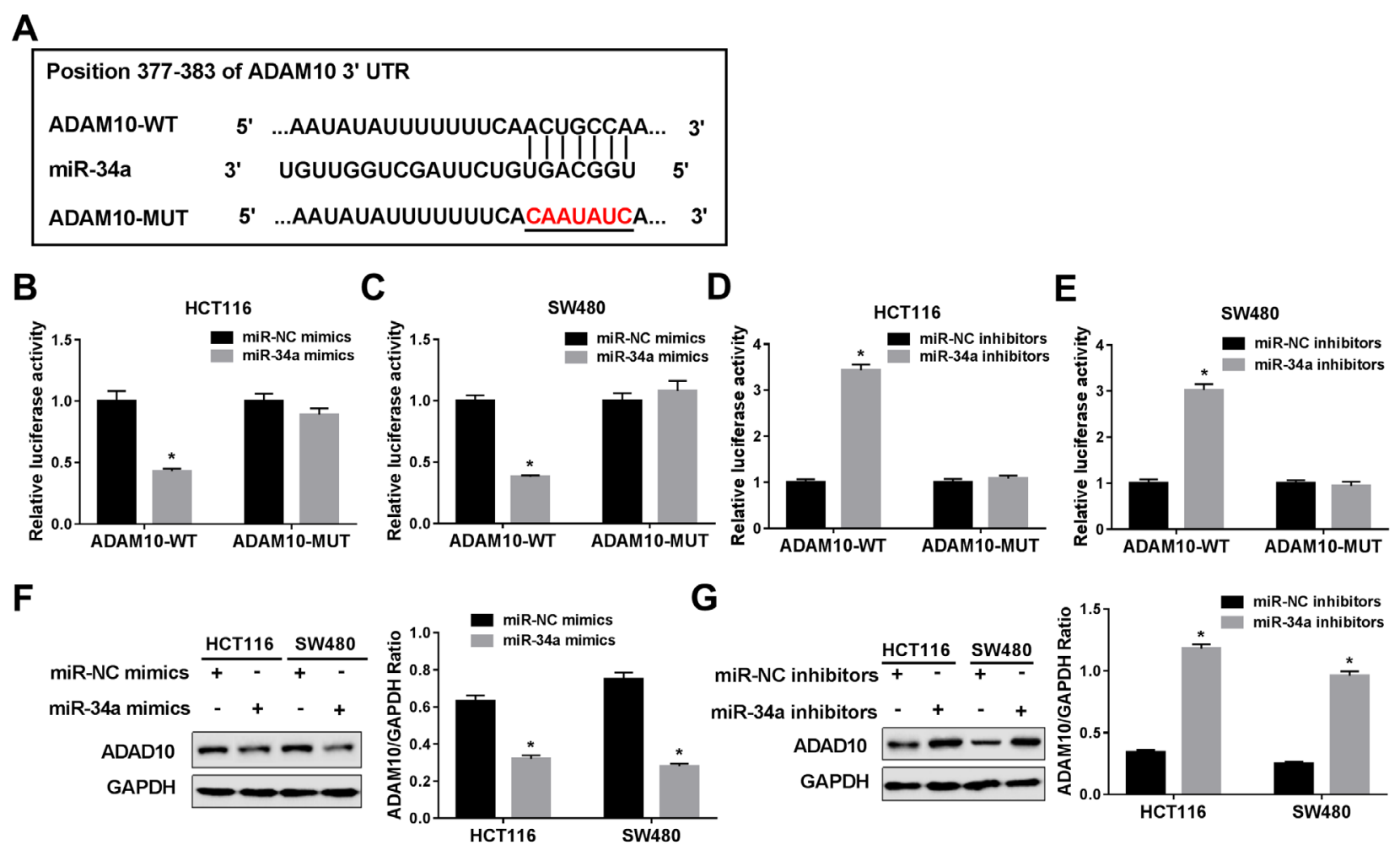

G
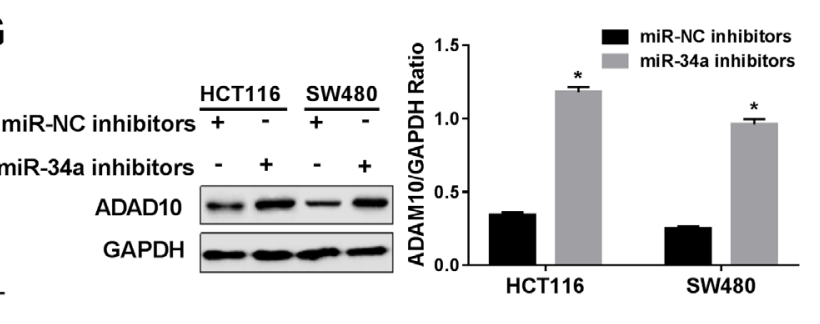

Figure 4. ADAM10 is a direct target of miR-34a and negatively regulated by miR-34a. A) The putative binding site and the mutant sites in of miR-34a on the 3'-UTR of ADAM10 mRNA are shown. B-E) HCT116 and SW480 cells were transfected with ADAM10-WT or ADAM10-MUT and miR-34a mimics or miR-NC mimics, followed by the measurement of luciferase activities at $48 \mathrm{~h}$ after transfection. F-G) The protein levels of ADAM10 in the two cell lines were tested by western blot after transfection with miR-34a mimics, miR-NC mimics, miR-34a inhibitors or miR-NC inhibitors. ${ }^{*} p<0.05$. 


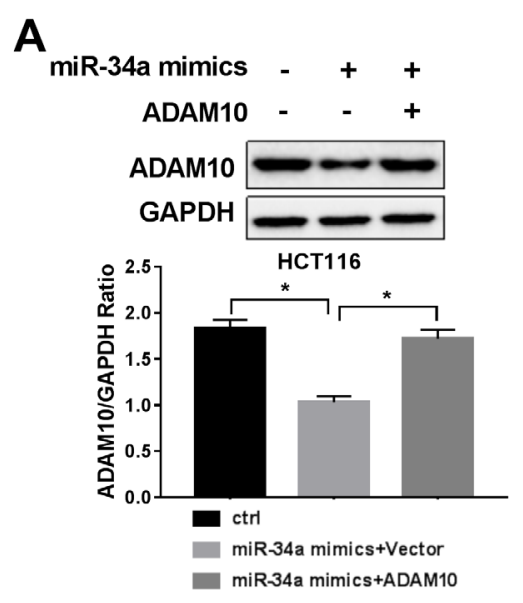

D

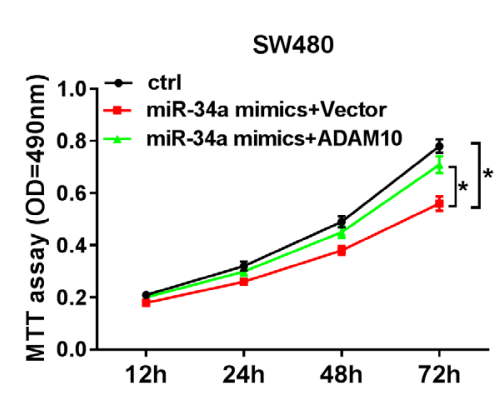

E
B
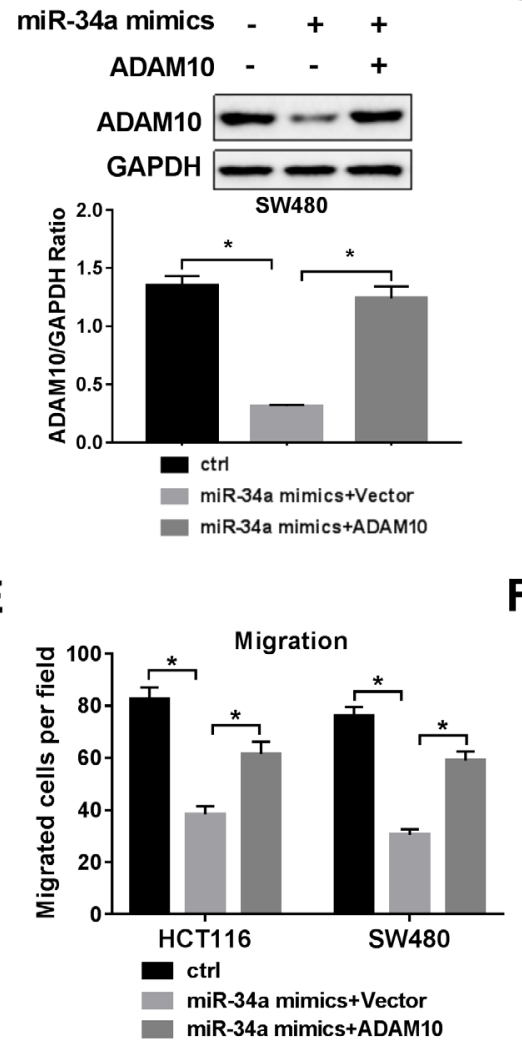

C

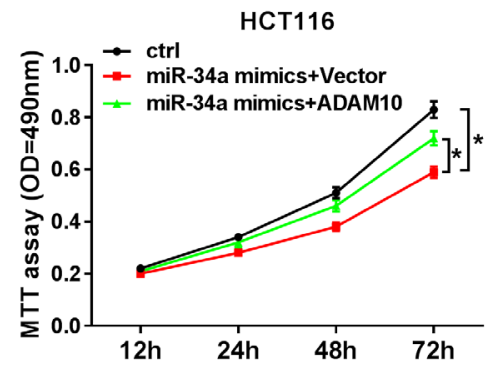

$\mathbf{F}$

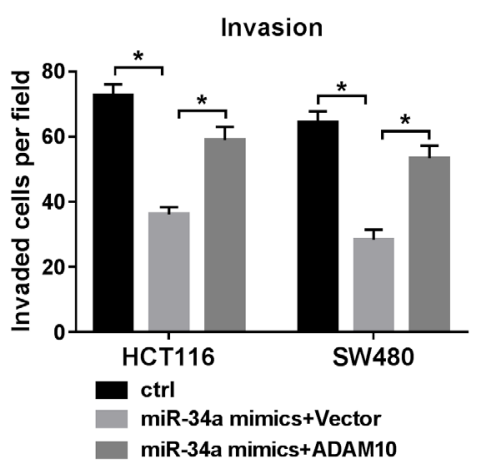

Figure 5. miR-34a inhibited the cell viability, migration and invasion abilities by targeting ADAM10. A and B) western blot assay revealed the protein level of ADAM10 in HCT116 and SW480 cells after co-transfection. C and D) MTT assay showed cell viability of HCT116 and SW480 cells after cotransfection. E and F) Transwell assay exhibited migration and invasion abilities of the two cell lines. ${ }^{\star} \mathrm{p}<0.05$, ctrl (without treatment).

was detected (Figures 4B-4E). The protein level of ADAM10 in HCT116 and SW480 cells was measured by western blot assay, and the results showed that the ADAM10 level was downregulated by miR-34a mimics (Figure $4 \mathrm{~F}$ ) and upregulated by miR-34a inhibitors (Figure $4 \mathrm{G}$ ).

miR-34a inhibited the cell viability, migration and invasion abilities by targeting ADAM10. In order to figure out the inhibitory mechanism of miR-34a on CRC cell lines, we conducted HCT116 and SW480 cells that miR-34a and ADAM10 upregulated meanwhile or miR-34a up-regulated alone by co-transfection. Western blot assay revealed that overexpression of ADAM10 recuperated the repression of ADAM10 expression by miR-34a mimics in the two cell lines (Figures 5A and 5B). Overexpression of ADAM10 also recovered the inhibitory effects of miR-34a mimics on cell viability, migration and invasion in both HCT116 and SW480 cells (Figures 5C-5F).

Upregulation of ADAM10 alleviated in part sevoflurane-mediated repression on CRC cell viability, migration and invasion. In order to further investigate the mechanism of sevoflurane in progress of CRC cells, we transfected HCT116 and SW480 cells with pcDNA3.1-ADAM10 to upregulate ADAM10 after treatment with 5.1\% sevoflurane. Results suggested that the upregulation of ADAM10 strikingly relieved the repression of expression of ADAM10 induced by sevoflurane in HCT116 and SW480 cells (Figures 6A and 6B). We also demonstrated that the upregulation of ADAM10 also partially recovered the suppressive influence on cell viability, migration and invasion in both HCT116 and SW480 cells (Figures 6C-6F).

\section{Discussion}

It is widely accepted that anesthetic agents and analgesia techniques have implications in cancer recurrence and metastases [26]. Therefore, it is crucial to set appropriate anesthetics and dose to avoid the migration and invasion of cancer cells and decrease the risk of cell diffusion during the surgical resection of tumors.

Previous studies demonstrated that sevoflurane exerts its inhibitory functions on the proliferation, migration and invasion of tumor cells by targeting miRNAs. In breast cancer, sevoflurane blocked the proliferation of MDA-MB231 cells by upregulating miR-203 [27]. Yi et al. validated that 
A

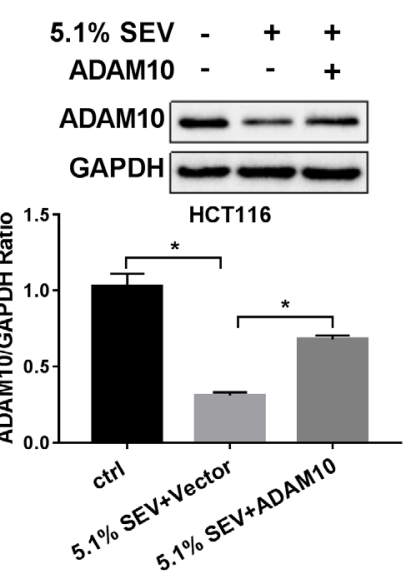

D

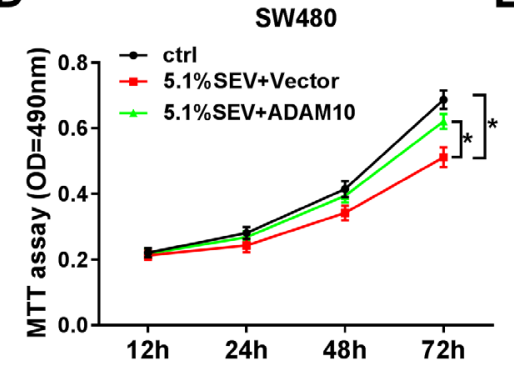

B

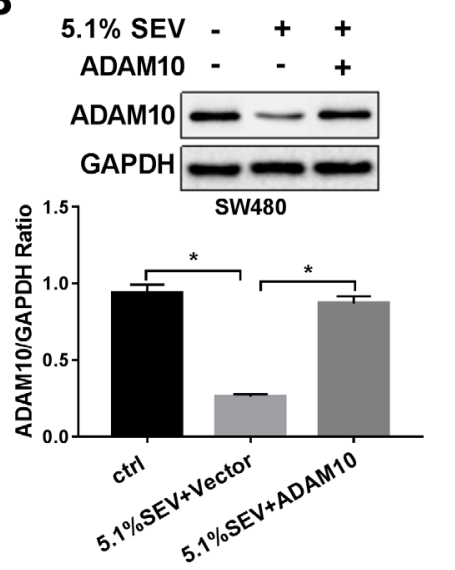

$\mathbf{E}$

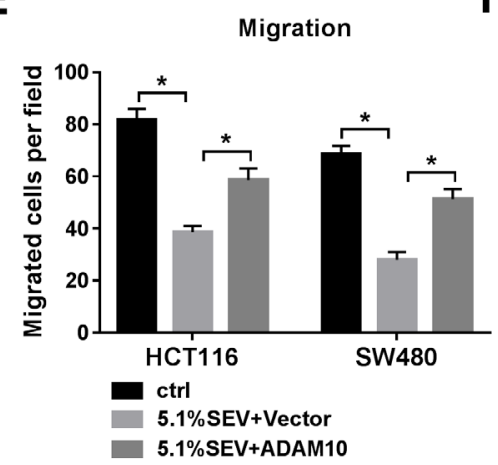

C

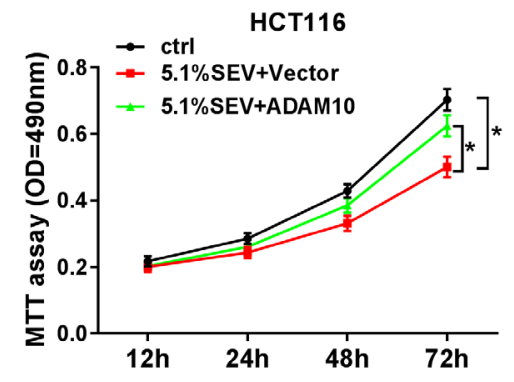

$\mathbf{F}$

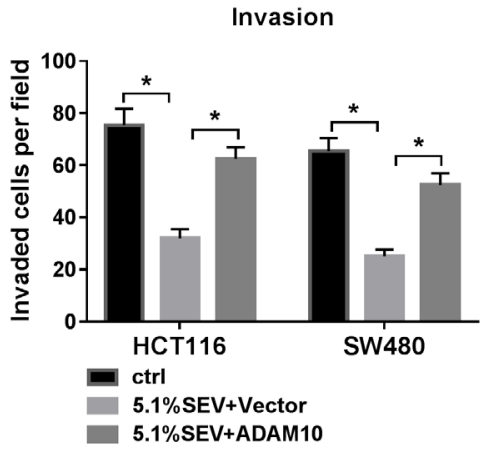

Figure 6. Upregulation of ADAM10 alleviated sevoflurane-mediated repression on CRC cell viability, migration and invasion. HCT116 and SW480 cells were transfected with pcDNA3.1 vector or pcDNA3.1-ADAM10 after treatment with 5.1\% sevoflurane. A-B) The protein level of ADAM10 in HCT116 and SW480 cells, C-D) cell viability of HCT116 and SW480 cells and E- F) migration and invasion cell numbers in HCT116 and SW480 cells are shown: ${ }^{*} \mathrm{p}<0.05$, ctrl (without treatment).

sevoflurane retarded migration and invasion of glioma cells by upregulating miR-637 [7]. In addition, sevoflurane could induce liver injury by mediating insulin-like growth factor 1 (IGF-1) expression via miR-214 [28]. In current study, strikingly inhibitory effects on the cell viability, migration and invasion abilities of CRC HCT116 and SW480 cells were observed. Then, efforts were made to clarify the molecular mechanism of inhibitory effects on CRC cells mediated by sevoflurane.

A former study indicated that overexpression of miR-34a led to cell cycle arrest and growth inhibition in prostate cancer [15]. Elevation of miR-34a expression potently suppressed cell migration and invasion of hepatocellular carcinoma cells in a c-Met-dependent manner [16]. It has been suggested that miR-34a could repress invasion and metastasis in gastric cancer by targeting Tgif2 [17]. Shi et al. demonstrated that ectopic expression of miR-34a obviously inhibited the cell growth rates and migration in human esophageal cancer ECA109 and TE1 cell lines in vitro [18]. In CRC, ectopic expression of miR-34a constrained the CRC cell growth in vitro and in vivo [19]. What's more, miR-34a could suppress CRC metastasis by targeting and regulating
Notch signaling pathway [29]. MiR-34a can also suppress the progression of CRC via targeting FMNL2 and E2F5 [30]. In the present study, we drew similar conclusion that gain of miR-34a apparently suppressed the cell viability, migration and invasion of HCT116 and SW480 cells. We also validated that the inhibitory effects of sevoflurane on CRC cells resulted from upregulating miR-34a.

Fogel et al. found that ADAM10 mediated the release of adhesion factor L1 and promoted cell migration and tumor metastasis in ovarian and uterine carcinomas [31]. It has been suggested that ADAM10 could lyse adhesion molecule CD44 facilitating cell migration and invasion of glioma U251 cells [32]. ADAM10 overexpression in colon cancer cells enhanced L1-CAM cleavage and triggered liver metastasis and elevated metastasis in colon cancer cells transfected with L1-CAM [33]. Literature data shows that ADAM10 has potential significance on mediation of tumor progress, metastasis and angiogenesis by regulating cell signaling pathways or cell adhesion [34]. Guo and his colleagues verified that ADAM10 was upregulated in non-small cell lung cancer (NSCLC) tissues, and ADAM10 enhanced the migration and invasion of NSCLC cells by 
activating the Notch1 signaling pathway [35]. It has been demonstrated that ADAM10 played important roles in the proliferation and metastasis of HCC cells and downregulation of ADAM10 significantly suppressed cell proliferation, migration and invasion of HCC cells in vitro [36]. In our study, ADAM10 was identified as a target gene of miR-34a and was negatively regulated by miR-34a. We found that miR-34a hindered the cell viability, migration and invasion of CRC cells by targeting ADAM10 and upregulation of ADAM10 could partially relieve the inhibitory effects of on CRC cells mediated by sevoflurane or miR-34a.

However, there are some limitations in this report. We only investigated the role of sevoflurane in vitro. The function of sevoflurane should be investigated in in vivo experiments in future. Besides, whether the type of anesthesia during surgery affects survival and time to progression in patients with early stages of cancer should be also investigated in future. We would provide more evidence for whether sevoflurane has an advantage in the prognosis of colon cancer via clinical data collection and statistical study.

In summary, we found the inhibitory effects on the cell viability, migration and invasion abilities of CRC HCT116 or SW480 cells. Then we tried to clarify mechanism of effects on CRC cells mediated by sevoflurane. We validated that sevoflurane exerts its repressive effects on CRC cells by targeting miR-34a. Next we confirmed the target interaction between miR-34a and ADAM10. Then we verified that gain of ADAM10 effectively rescued the suppressive influence of sevoflurane and miR-34a, indicating that sevoflurane inhibits the migration and invasion of colorectal cancer cells by regulating microRNA-34a/ADAM10 axis. In a word, we observed a novel regulation axis of sevoflurane on CRC progression, which may supply references for clinical surgery or treatment of CRC.

\section{References}

[1] CENTER MM, JEMAL A, SMITH RA, WARD E. Worldwide variations in colorectal cancer. CA Cancer J Clin 2009; 59: 366-378. https://doi.org/10.3322/caac.20038

[2] SUNG JJ, NG SC, CHAN FK, CHIU HM, KIM HS et al. An updated Asia Pacific Consensus Recommendations on colorectal cancer screening. Gut 2015; 64: 121-132. https:// doi.org/10.1136/gutjnl-2013-306503

[3] MORIARITY A, O'SULLIVAN J, KENNEDY J, MEHIGAN B, MCCORMICK P. Current targeted therapies in the treatment of advanced colorectal cancer: a review. Ther Adv Med Oncol 2016; 8: 276-293. https://doi. org/10.1177/1758834016646734

[4] KVOLIK S, DOBROSEVIC B, MARCZI S, PRLIC L, GLAVAS-OBROVAC L. Different apoptosis ratios and gene expressions in two human cell lines after sevoflurane anaesthesia. Acta Anaesthesiol Scand 2009; 53: 1192-1199. https:// doi.org/10.1111/j.1399-6576.2009.02036.x
[5] KVOLIK S, GLAVAS-OBROVAC L, BARES V, KARNER I. Effects of inhalation anesthetics halothane, sevoflurane, and isoflurane on human cell lines. Life Sci 2005; 77: 2369-2383. https://doi.org/10.1016/j.lfs.20 04.12.052

[6] LIANG H, GU M, YANG C, WANG H, WEN X et al. Sevoflurane inhibits invasion and migration of lung cancer cells by inactivating the p38 MAPK signaling pathway. J Anesth 2012; 26: 381-392. https://doi.org/10.1007/s00540-0111317-y

[7] YI W, LI D, GUO Y, ZHANG Y, HUANG B et al. Sevoflurane inhibits the migration and invasion of glioma cells by upregulating microRNA-637. Int J Mol Med 2016; 38: 1857-1863. https://doi.org/10.3892/ijmm.2016.2797

[8] KLOOSTERMAN WP, PLASTERK RH. The diverse functions of microRNAs in animal development and disease. Dev Cell 2006; 11: 441-450. https://doi.org/10.1016/j.devcel.2006.09.009

[9] DI LEVA G, GAROFAlO M, CROCE CM. MicroRNAs in cancer. Annu Rev Pathol 2014; 9: 287-314. https://doi. org/10.1146/annurev-pathol-012513-104715

[10] FAN J, ZHANG W, WU Y, WAN P, GUO Q et al. miR124 inhibits cell growth through targeting IQGAP1 in colorectal cancer. Mol Med Rep 2018; 18: 5270-5278. https://doi. org/10.3892/mmr.2018.9518

[11] WANG YX, ZHU HF, ZHANG ZY, REN F, HU YH. MiR384 inhibits the proliferation of colorectal cancer by targeting AKT3. Cancer Cell Int 2018; 18: 124. https://doi. org/10.1186/s12935-018-0628-6

[12] ZHANG Y, WANG X, WANG Z, TANG H, FAN H et al. miR182 promotes cell growth and invasion by targeting forkhead box F2 transcription factor in colorectal cancer. Oncol Rep 2015; 33: 2592-2598. https://doi.org/10.3892/or.2015.3833

[13] CHEN E, LI Q, WANG H, YANG F, MIN L et al. MiR-92a promotes tumorigenesis of colorectal cancer, a transcriptomic and functional based study. Biomed Pharmacother 2018; 106: 1370-1377. https://doi.org/10.1016/j.biopha.2018.07.098

[14] HERMEKING H. The miR-34 family in cancer and apoptosis. Cell Death Differ 2010; 17: 193-199. https://doi. org/10.1038/cdd.2009.56

[15] FUJITA Y, KOJIMA K, HAMADA N, OHHASHI R, AKAO $\mathrm{Y}$ et al. Effects of miR-34a on cell growth and chemoresistance in prostate cancer PC3 cells. Biochem Biophys Res Commun 2008; 377: 114-119. https://doi.org/10.1016/j. bbrc.2008.09.086

[16] LI N, FU H, TIE Y, HU Z, KONG W et al. miR-34a inhibits migration and invasion by down-regulation of c-Met expression in human hepatocellular carcinoma cells. Cancer Lett 2009; 275: 44-53. https://doi.org/10.1016/j.canlet.2008.09.035

[17] HU Y, PU Q, CUI B, LIN J. MicroRNA-34a inhibits tumor invasion and metastasis in gastric cancer by targeting Tgif2. Int J Clin Exp Pathol 2015; 8: 8921-8928.

[18] SHI H, ZHOU S, LIU J, ZHU J, XUE J et al. miR-34a inhibits the in vitro cell proliferation and migration in human esophageal cancer. Pathol Res Pract 2016; 212: 444-449. https:// doi.org/10.1016/j.prp.2016.02.019 
[19] ZHANG Q, WANG J, LI N, LIU Z, CHEN Z et al. miR-34a increases the sensitivity of colorectal cancer cells to 5 -fluorouracil in vitro and in vivo. Am J Cancer Res 2018; 8: 280-290.

[20] EDWARDS DR, HANDSLEY MM, PENNINGTON CJ. The ADAM metalloproteinases. Mol Aspects Med 2008; 29: 258289. https://doi.org/10.1016/j.mam.2008.08.001

[21] BLOBEL CP. ADAMs: key components in EGFR signalling and development. Nat Rev Mol Cell Biol 2005; 6: 32-43. https://doi.org/10.1038/nrm1548

[22] DUFFY MJ, MCKIERNAN E, O'DONOVAN N, MCGOWAN PM. Role of ADAMs in cancer formation and progression. Clin Cancer Res 2009; 15: 1140-1144. https://doi. org/10.1158/1078-0432.ccr-08-1585

[23] YONEYAMA T, GORRY M, SOBO-VUJANOVIC A, LIN Y, VUJANOVIC L et al. ADAM10 Sheddase Activity is a Potential Lung-Cancer Biomarker. J Cancer 2018; 9: 2559-2570. https://doi.org/10.7150/jca.24601

[24] SHIU JS, HSIEH MJ, CHIOU HL, WANG HL, YEH CB et al. Impact of ADAM10 gene polymorphisms on hepatocellular carcinoma development and clinical characteristics. Int J Med Sci 2018; 15: 1334-1340. https://doi.org/10.7150/ ijms.27059

[25] WANG L, WANG T, GU JQ, SU HB. Volatile anesthetic sevoflurane suppresses lung cancer cells and miRNA interference in lung cancer cells. Onco Targets Ther 2018; 11: 5689-5693. https://doi.org/10.2147/ott.s171672

[26] NIWA H, ROWBOTHAM DJ, LAMBERT DG, BUGGY DJ. Can anesthetic techniques or drugs affect cancer recurrence in patients undergoing cancer surgery? J Anesth 2013, 27: 731-741. https://doi: 10.1007/s00540-013-1615-7

[27] LIU J, YANG L, GUO X, JIN G, WANG Q et al. Sevoflurane suppresses proliferation by upregulating microRNA-203 in breast cancer cells. Mol Med Rep 2018; 18: 455-460. https:// doi.org/10.3892/mmr.2018.8949

[28] MA L, ZHAO J, XIE X. Sevoflurane induces liver injury by modulating the expression of insulin-like growth factor 1 via miR-214. J Cell Physiol 2018; 233: 6742-6749. https://doi. org/10.1002/jcp.26382
[29] ZHANG X, AI F, LI X, TIAN L, WANG X et al. MicroRNA34a suppresses colorectal cancer metastasis by regulating Notch signaling. Oncol Lett 2017; 14: 2325-2333. https://doi. org/10.3892/ol.2017.6444

[30] LU G, SUN Y, AN S, XIN S, REN X et al. MicroRNA-34a targets FMNL2 and E2F5 and suppresses the progression of colorectal cancer. Exp Mol Pathol 2015; 99: 173-179. https:// doi.org/10.1016/j.yexmp.2015.06.014

[31] FOGEL M, GUTWEIN P, MECHTERSHEIMER S, RIEDLE $\mathrm{S}$, STOECK A et al. L1 expression as a predictor of progression and survival in patients with uterine and ovarian carcinomas. Lancet 2003; 362: 869-875. https://doi.org/10.1016/ s0140-6736(03)14342-5

[32] MURAI T, MIYAZAKI Y, NISHINAKAMURA H, SUGAHARA KN, MIYAUCHI T et al. Engagement of CD44 promotes Rac activation and CD44 cleavage during tumor cell migration. J Biol Chem 2004; 279: 4541-4550. https://doi. org/10.1074/jbc.M307356200

[33] GAVERT N, SHEFFER M, RAVEH S, SPADERNA S, SHTUTMAN M et al. Expression of L1-CAM and ADAM10 in human colon cancer cells induces metastasis. Cancer Res 2007; 67: 7703-7712. https://doi.org/10.1158/0008-5472. can-07-0991

[34] SAFTIG P, REISS K. The "A Disintegrin And Metalloproteases" ADAM10 and ADAM17: novel drug targets with therapeutic potential? Eur J Cell Biol 2011; 90: 527-535. https:// doi.org/10.1016/j.ejcb.2010.11.005

[35] GUO J, HE L, YUAN P, WANG P, LU Y et al. ADAM10 overexpression in human non-small cell lung cancer correlates with cell migration and invasion through the activation of the Notch1 signaling pathway. Oncol Rep 2012; 28: 17091718. https://doi.org/10.3892/or.2012.2003

[36] LIU S, ZHANG W, LIU K, JI B, WANG G. Silencing ADAM10 inhibits the in vitro and in vivo growth of hepatocellular carcinoma cancer cells. Mol Med Rep 2015; 11 : 597-602. https://doi.org/10.3892/mmr.2014.2652 